\title{
De- and repolarization mechanism of flagellar morphogenesis during a bacterial cell cycle
}

\author{
Nicole J. Davis, ${ }^{1,5}$ Yaniv Cohen, ${ }^{2,5,6}$ Stefano Sanselicio, ${ }^{3,6}$ Coralie Fumeaux, ${ }^{3}$ Shogo Ozaki, ${ }^{2}$ \\ Jennifer Luciano, ${ }^{2}$ Ricardo C. Guerrero-Ferreira, ${ }^{4}$ Elizabeth R. Wright, ${ }^{4}$ Urs Jenal, ${ }^{2,7}$ \\ and Patrick. H. Viollier ${ }^{1,3,7}$ \\ ${ }^{1}$ Department of Molecular Biology and Microbiology, School of Medicine, Case Western Reserve University, Cleveland, Ohio \\ 44106, USA; ${ }^{2}$ Biozentrum of the University of Basel, 4056 Basel, Switzerland; ${ }^{3}$ Department Microbiology and Molecular \\ Medicine, Institute of Genetics and Genomics in Geneva (iGE3), Faculty of Medicine/Centre Médical Universitaire, University \\ of Geneva, 1211 Genève 4, Switzerland; ${ }^{4}$ Division of Pediatric Infectious Diseases, Department of Pediatrics, Emory University \\ School of Medicine, Children's Healthcare of Atlanta, Atlanta, Georgia 30322, USA
}

Eukaryotic morphogenesis is seeded with the establishment and subsequent amplification of polarity cues at key times during the cell cycle, often using (cyclic) nucleotide signals. We discovered that flagellum de- and repolarization in the model prokaryote Caulobacter crescentus is precisely orchestrated through at least three spatiotemporal mechanisms integrated at TipF. We show that TipF is a cell cycle-regulated receptor for the second messenger-bis-( $\left.3^{\prime}-5^{\prime}\right)$-cyclic dimeric guanosine monophosphate (c-di-GMP) - that perceives and transduces this signal through the degenerate c-di-GMP phosphodiesterase (EAL) domain to nucleate polar flagellum biogenesis. Once c-di-GMP levels rise at the G1 $\rightarrow \mathrm{S}$ transition, TipF is activated, stabilized, and polarized, enabling the recruitment of downstream effectors, including flagellar switch proteins and the PflI positioning factor, at a preselected pole harboring the TipN landmark. These c-di-GMP-dependent events are coordinated with the onset of tipF transcription in early $S$ phase and together enable the correct establishment and robust amplification of TipF-dependent polarization early in the cell cycle. Importantly, these mechanisms also govern the timely removal of TipF at cell division coincident with the drop in c-di-GMP levels, thereby resetting the flagellar polarization state in the next cell cycle after a preprogrammed period during which motility must be suspended.

[Keywords: asymmetric division; c-di-GMP; Caulobacter; cell cycle; flagellum; morphogenesis; polarity]

Supplemental material is available for this article.

Received May 22, 2013; revised version accepted August 23, 2013.

Cell polarity is fundamental to intracellular organization and morphogenesis in all forms of life, yet little is known about how polarity-dependent cues are implemented, reinforced, and removed during the bacterial cell cycle. Eukaryotic cells interpret (cyclic) nucleotide signals to regulate polarity-dependent processes via effectors such as kinases and/or GTPases dictated in response to spatiotemporal cues provided by prepositioned "landmark" proteins and cell cycle regulators (Slaughter et al. 2009; Charest and Firtel 2010; St Johnston and Ahringer 2010; Amato et al. 2011; Ji and Tulin 2012). Here we unearth a polarized cyclic nucleotide signaling pathway cued by bis- $\left(3^{\prime}-5^{\prime}\right)$-cyclic dimeric guanosine monophosphate

\footnotetext{
${ }^{5}$ These authors contributed equally to this work.

${ }^{6}$ These authors contributed equally to this work.

${ }^{7}$ Corresponding authors

E-mail patrick.viollier@unige.ch

E-mail urs.jenal@unibas.ch

Article is online at http://www.genesdev.org/cgi/doi/10.1101/gad.222679.113.
}

(c-di-GMP) that first triggers and reinforces and later resets flagellar polarity during a bacterial cell cycle.

The Gram-negative $\alpha$-proteobacterium Caulobacter crescentus (henceforth, Caulobacter) is an ideal model system to study how polarization is coordinated with cell cycle progression. At the predivisional cell stage, Caulobacter is overtly polarized, bearing a cylindrical extension of the cell envelope (the stalk) at the old pole and a newly assembled flagellum whose rotation is activated at cytokinesis at the opposite pole (Tsokos and Laub 2012). Cytokinesis yields a motile swarmer cell that resides in a G1-like, nonreplicative state and a dividing stalked cell engaged in S phase (Fig. 1A). Two master transcriptional regulators of the cell cycle, CtrA and GcrA, reinforce the transcriptional program at sequential stages of the cell cycle (Quon et al. 1996; Laub et al. 2000; Holtzendorff et al. 2004). CtrA is present in G1 phase, proteolytically removed during the $\mathrm{G} 1 \rightarrow \mathrm{S}$ transition, and reappears later in $S$ phase (Figs. 1A, 7D [below]). In 
A

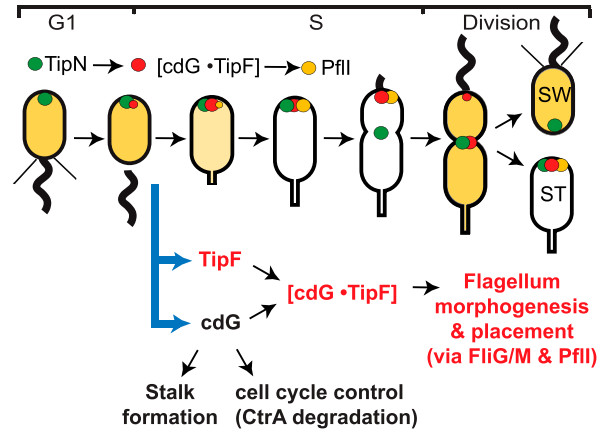

D

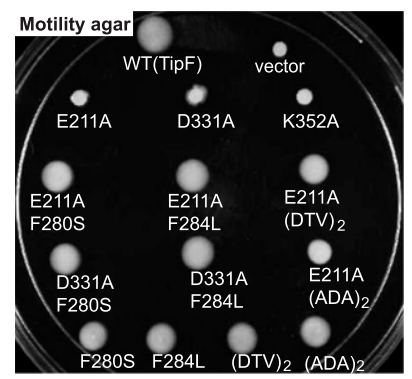

B

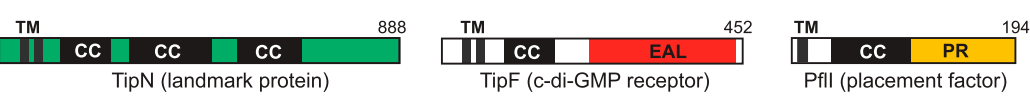

C

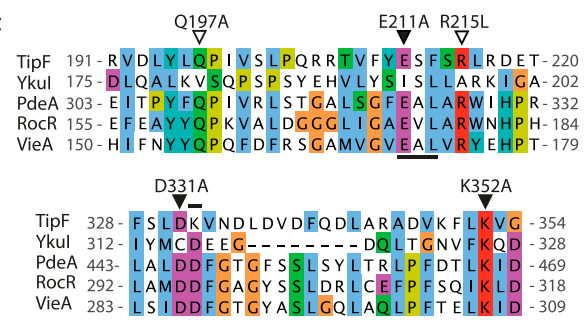

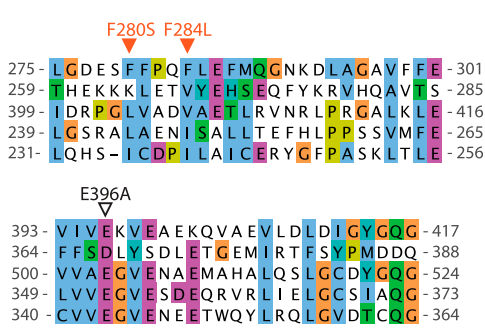

Figure 1. Localization of TipN, TipF, and PflI to the flagellated pole. (A) Dynamic protein localization of TipN, TipF, and PflI and its coordination with other cell cycle events in C. crescentus. TipN (green) localized at the nonflagellated pole recruits newly synthesized TipF (red), which in turn recruits the PflI flagellar positioning protein (yellow). Thick blue arrows indicate the synthesis of TipF (by GcrA) and c-di-GMP. (B) Domain organization of TipN, TipF, and PflI. Shown are the predicted transmembrane domains (TM; gray), coiled-coil domains (CC; black), PDE domain (EAL; red), and proline-rich domain ( $\mathrm{PR}$; yellow). $(C)$ Alignment of the EAL domains from TipF and the c-di-GMP PDEs PdeA of $C$. crescentus, VieA of Vibrio cholerae, RocR of Pseudomonas aeruginosa, and YkuI of Bacillus subtilis. Key residues that are predicted to be required for PDE activity (empty arrowheads) or required for c-di-GMP binding (filled black) are marked. Residues that bypass the requirement of E211 or D331 for motility (red triangles), the defining EAL motif, and the K332 residue of the conserved Asp-Asp loop (underlined) are indicated. (D) Motility assay of $\triangle t i p F$ mutants harboring plasmids encoding wild-type (WT) or single- or double-mutant TipF. DTV and ADA denote the triple duplications of DTV or ADA residues at positions 121-124 or 128-130, respectively. Overnight cultures were spotted on PYE swarm agar plates and incubated for $60 \mathrm{~h}$ at $30^{\circ} \mathrm{C}$. Compact swarms indicate the motility defect caused by mutations in the c-di-GMP-binding site, whereas the suppressive mutations yield diffuse and enlarged swarms. contrast, GcrA accumulates during the G1 $\rightarrow$ S transition, inducing the synthesis of CtrA along with polarity and other cell cycle proteins (McAdams and Shapiro 2011), and is then eliminated from G1 progeny cells along with c-di-GMP (Paul et al. 2008; Christen et al. 2010).

During the G1 $\rightarrow \mathrm{S}$ transition, the flagellum is shed to permit cells to immobilize to surfaces using an adhesive polar structure. The flagellum is then resynthesized at the new pole in late $S$ phase following this preprogrammed nonmotile period. CtrA induces the synthesis of early flagellar structural constituents, including the FliF MS ring protein and two components of the switch complex (FliG and FliM) (see Fig. 3A, below). The newly synthesized flagellar parts are assembled from the inside of the cell outward, first with an organizational platform in the inner membrane (the MS ring) to which the switch complex on the cytoplasmic side of the membrane is tethered (Chevance and Hughes 2008; Brown et al. 2011). The assembly of subsequent structures follows suit, ultimately ending with the elaboration of the external parts, such as the hook and the flagellar filament.

Superimposed on these temporal patterns are spatial cues that guide flagellar proteins to the appropriate site of assembly at the new cell pole opposite the stalk (Fig. 1A). The new cell pole harbors TipN, a polytopic coiled-coil protein that is deposited at the new pole as it is generated during cytokinesis (Huitema et al. 2006; Lam et al. 2006). At cytokinesis, TipN redistributes to the division plane from the poles, ensuring that cell polarity cues are avail- able for the flagellar polarization in the next cell cycle. Indeed, flagella are mispositioned in the absence of tipN, indicating that TipN is required for the proper placement, but not the assembly, of the flagellum (Huitema et al. 2006; Lam et al. 2006). A flagellar misplacement defect has also been observed in mutants lacking the PflI positioning factor, a bitopic membrane protein with a prolinerich domain at the $\mathrm{C}$ terminus facing the cytoplasm that is localized to the future flagellated pole before the synthesis of early flagellar components (Obuchowski and Jacobs-Wagner 2008). While the spatial relationship between TipN and PflI remains unexplored, TipN recruits TipF, a polytopic membrane protein that positively regulates flagellum biogenesis (Huitema et al. 2006). In the absence of TipN, TipF localizes to sites of misplaced flagella, raising the possibility that mislocalized TipF is responsible for the flagellar misplacement phenotype of a tipN mutant. This is supported by the observation that tipF deletion mutants lack external flagellar structures such as the hook and filament (Huitema et al. 2006).

TipF features a C-terminal and cytoplasmic EAL (also known as DUF2) domain that, in related proteins, confers c-di-GMP-specific phosphodiesterase (PDE) activity (Hengge 2009; Schirmer and Jenal 2009; Boyd and O'Toole 2012). The levels of ci-di-GMP are modulated by diguanylate cyclases (DGCs) that synthesize c-di-GMP from GTP and PDEs that hydrolyze c-di-GMP into linear pGpG. In Caulobacter, multiple DGCs and PDEs determine c-di-GMP levels during the cell cycle to promote develop- 
ment and cell cycle progression (Paul et al. 2004, 2008; Abel et al. 2011). c-di-GMP levels are low in G1 cells but peak at the G1 $\rightarrow$ S transition and decline thereafter (Paul et al. 2008; Christen et al. 2010). While c-di-GMP binds to various receptor proteins to curb flagellar rotation (Wolfe and Visick 2008; Boehm et al. 2010; Fang and Gomelsky 2010; Paul et al. 2010), the spatiotemporal mechanism underling c-di-GMP-induced flagellum morphogenesis has not been resolved.

Here we show that c-di-GMP controls the activation, polarization, and stabilization of the flagellar regulator TipF (Huitema et al. 2006). We found that TipF is a receptor for c-di-GMP that peaks at the G1 $\rightarrow \mathrm{S}$ transition and show that TipF*c-di-GMP seeds polar flagellar assembly by recruiting the PflI placement factor (Obuchowski and Jacobs-Wagner 2008) and components of the flagellar switch into a complex with TipN, a landmark protein that is prepositioned at the newborn pole (Huitema et al. 2006; Lam et al. 2006). Importantly, we found that c-diGMP-induced stabilization and polarization of TipF is amplified with the coordinated transcription of the tipF gene and thus is tuned to other c-di-GMP-dependent developmental events that occur at the G1 $\rightarrow$ S transition (Paul et al. 2004, 2008; Duerig et al. 2009).

\section{Results}

\section{c-di-GMP binds and activates TipF}

Initial hints into the mechanism of TipF-mediated flagellum biogenesis came from comparisons of the TipF primary structure with known PDEs. TipF features a Lys at position 332 instead of an Asp in related proteins (Fig.
1C), and a Glu-Ser-Phe (ESF, residues 211-213) triplet replaces the defining Glu-Ala-Leu (EAL) motif. The highly conserved Asp331-Asp332 motif is a hallmark of active PDEs, with both residues being involved in coordinating the two metal ions in the catalytic center (Barends et al. 2009). To explore whether the sequence conservation reflects a functional requirement for the residues at these positions, we engineered alanine mutations at several conserved positions implicated or not in coordinating the cofactor $\left(\mathrm{Mg}^{2+}\right.$ or $\left.\mathrm{Mn}^{2+}\right)$, the substrate, and/or the nucleophile (E211A, D331A, and K352A) (Fig. 1C, filled triangles). Neither of these mutant proteins could support TipF function (motility on $0.3 \%$ soft agar plates) when expressed in a $\Delta t i p F$ background from $P_{x y l}$ on a low-copy plasmid, indicating that these residues play a key role in TipF function (Fig. 1D).

To test whether TipF is an active PDE, we purified the soluble hexa-histidine $\left(\mathrm{His}_{6}\right)$-tagged form of TipF lacking the first 115 residues, including the two predicted membrane-spanning segments, and the E211A mutant derivative from Escherichia coli. Circular dichroism (CD) spectra suggested that the secondary structures of purified TipF wild type and E211A mutant were unchanged (Supplemental Fig. S1A). We then assayed both proteins for PDE activity by high-pressure liquid chromatography (HPLC) analysis with the c-di-GMP substrate (Fig. 2A). No cleavage of c-di-GMP to the linear form $\mathrm{pGpG}$ was observed with either wild-type TipF or the E211A mutant (Fig. 2A, right panel) even after extended incubation times of up to $24 \mathrm{~h}$ (Supplemental Fig. S1B,C). In contrast, the control PDE YahA from E. coli converted all of the c-di-GMP to pGpG in $<5$ min (Fig. 2A, left panel).
A
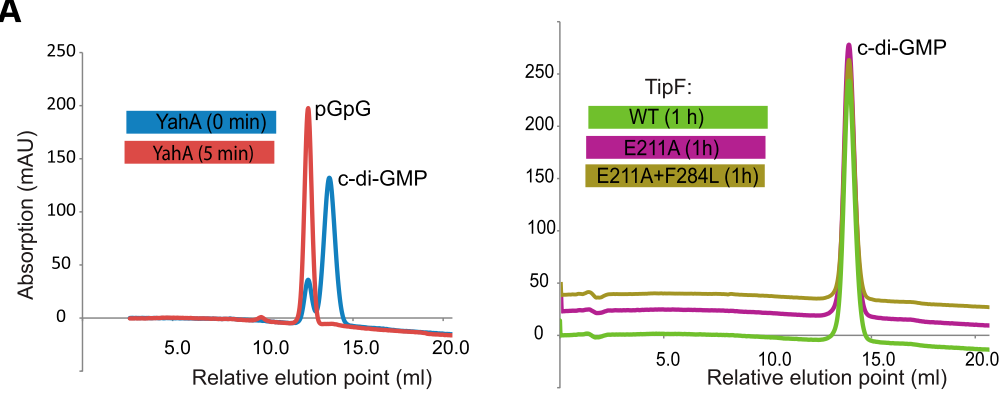

B
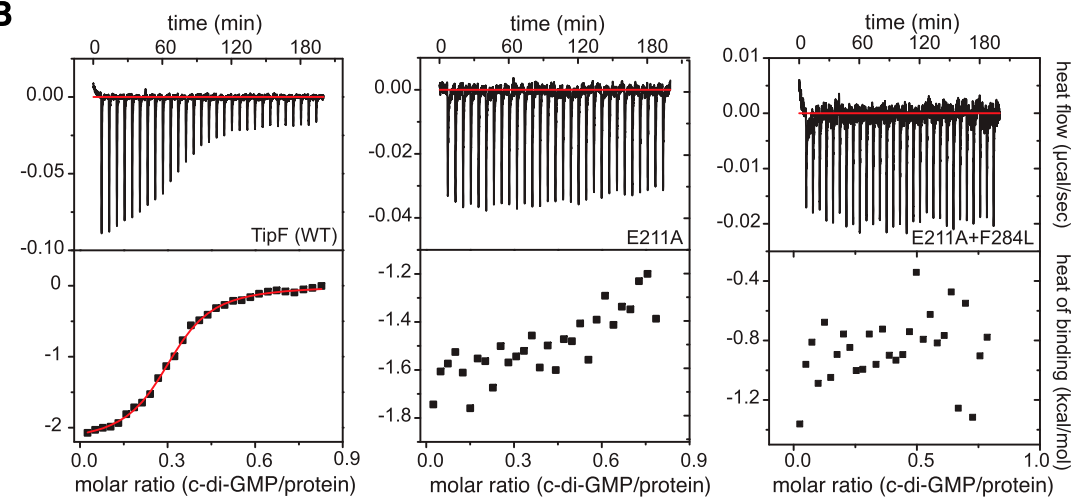

Figure 2. TipF is enzymatically inactive but binds c-di-GMP. (A) c-di-GMP hydrolytic activity is not detected in recombinant (soluble) TipF but is in the control PDE YahA. (Left panel) Purified proteins $(1 \mu \mathrm{M})$ were incubated with c-di-GMP for $5 \mathrm{~min}$ and then separated on a ResourceQ column to observe the cleavage product pGpG. No pGpG was detected after $1 \mathrm{~h}$ of incubation with wildtype (WT) or mutant (E211A or E211A/F284L) TipF with c-di-GMP. (B) ITC experiments showing that c-di-GMP binds to the soluble portion of wild-type TipF but not to the mutant derivatives E211A or E211A/F284L. The top panels show the raw ITC data curves collected at $25^{\circ} \mathrm{C}$ in binding buffer $(50 \mathrm{mM}$ Tris/HCl, $50 \mathrm{mM} \mathrm{NaCl}$ at $\mathrm{pH} 8.0$ ). The bottom panels show the integrated titration peaks fitted to a one-site binding model (solid line). The average dissociation constant $\left(\mathrm{K}_{\mathrm{D}}\right)$ of TipF to c-di-GMP was estimated at 0.4 $( \pm 0.2 \mu \mathrm{M})$, the stoichiometry of binding $(n)$ was estimated at $0.35( \pm 0.1)$, and the enthalpy of reaction was estimated at -2.1 $( \pm 0.3 \mathrm{kcal} / \mathrm{mol})$. 
In the absence of detectable PDE activity, we asked whether wild-type TipF or the E211A mutant can bind c-di-GMP using an isothermal titration calorimetry (ITC)-based binding assay (Fig. 2B). Successive injections of $10 \mu \mathrm{L}$ of a $118 \mu \mathrm{M}$ solution of c-di-GMP solution into the ITC reaction chamber containing $32.5 \mu \mathrm{M}$ wild-type TipF was accompanied by the characteristic heat release, reflecting specific and high-affinity binding of c-di-GMP (Fig. 2B, left panel). The resulting integrated titration peaks were fitted to a sigmoidal enthalpy curve, and a dissociation constant $\left(\mathrm{K}_{\mathrm{D}}\right)$ of $0.4( \pm 0.2 \mu \mathrm{M})$ for c-di-GMP was derived for wild-type TipF. In contrast, only background (nonspecific) heat release was observed when an equimolar amount of TipF (E211A) was injected into the chamber (Fig. 2B, middle panel [note the different scales in the panels]). Together, these results demonstrate that TipF does not have PDE activity under the conditions tested and that wild-type TipF, but not the E211A derivative, binds c-di-GMP with high affinity. On the basis of these results, we hypothesize that c-di-GMP binding is necessary for TipF to signal motility and that the E211A mutant is nonfunctional because it can no longer bind and be activated by c-di-GMP. In support of this view, we found that depleting Caulobacter cells of c-di-GMP phenocopies the localization and flagellar assembly defect caused by the absence TipF or the "c-di-GMPblind" E211A mutant (Fig. 6E, below).
If TipF is activated without hydrolyzing c-di-GMP, then mutations that are known to specifically cripple catalytic activity of PDEs, but not c-di-GMP binding, should not affect TipF function. To test this prediction, we engineered mutants Q197A, R215L, and E396A (Fig. 1C, open triangles) and found that the mutant proteins $\mathrm{R} 215 \mathrm{~L}$ and E396A confer motility in $\Delta$ tipF cells indistinguishable from that of wild-type TipF, while Q197A also supported motility but with reduced efficiency (data not shown). Taken together, we conclude that TipF binds c-di-GMP and that binding is required for flagellar functions.

\section{TipF acts early in polar flagellum assembly}

Since previous transmission electron micrographs showed the external structures, the flagellar hook, and the filament (Fig. 3A) to be missing from $\triangle$ tipF cells (Huitema et al. 2006), we wondered whether the assembly of internal flagellar (sub)structures also requires TipF. To this end, we conducted electron cryotomography (ECT) of wildtype and $\triangle$ tipF cells by ECT (Fig. 3B; Supplemental Fig. $\mathrm{S} 2 \mathrm{~A}, \mathrm{~B}$; see the Supplemental Movies), a high-resolution imaging method that preserves cellular structures in their native (cryogenically vitrified) state and reveals them owing to the natural contrast from electron density. We subjected two-dimensional (2D) projections of three-
A

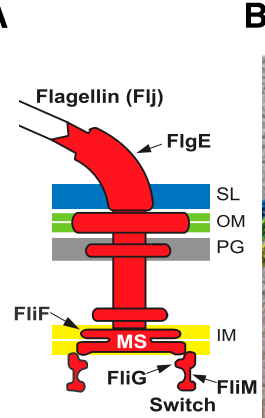

C

$\frac{\mathrm{pP}_{\text {van-fliM-GFP plasmid in: }}}{\text { fliM::Tn5 } \Delta \text { tipF; fliM::Tn5 } \Delta \text { fliG; fliM::Tn5 }}$

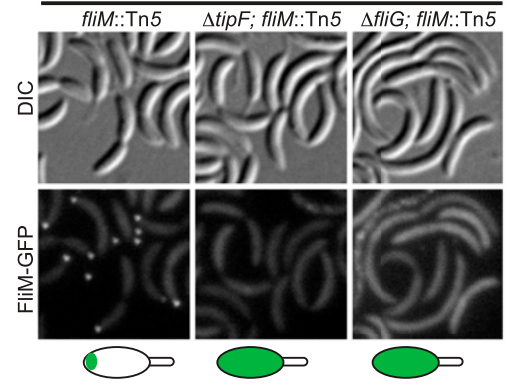

E

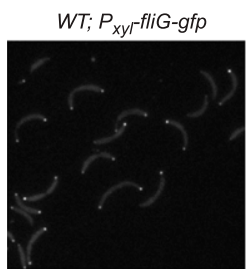

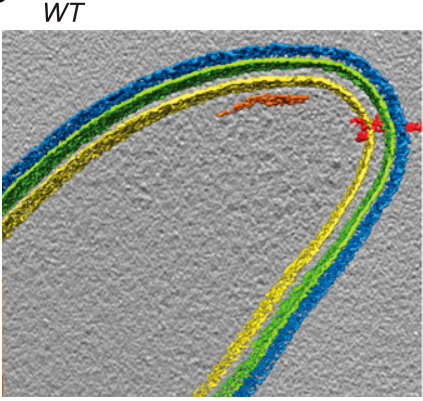

D

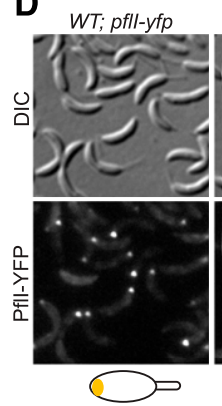

$\Delta t i p F ; P_{x y l-f l i G-g f p}$

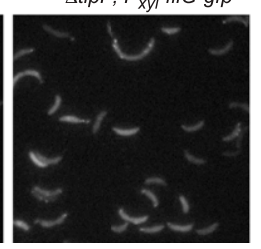

$\mathbf{F}$
$\Delta t i p F$

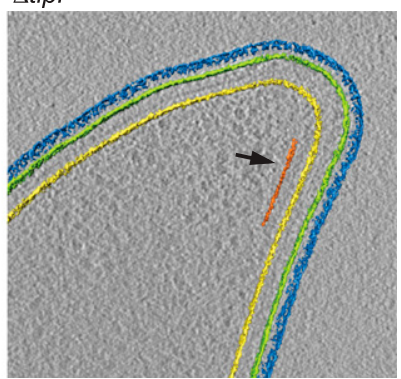

$\Delta t i p F ; p f l l-y f p$

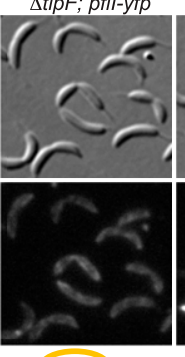

$\infty$

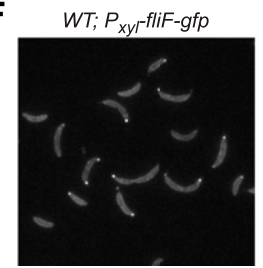

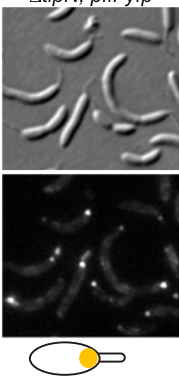

$\Delta$ tipF; $P_{x y l}$ fliF-gfp

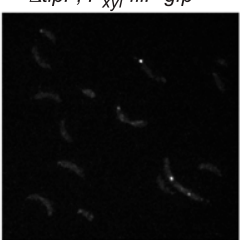

$\Delta t i p N ;$ pfl-yfp

Figure 3. TipF mediates the localization of flagellar proteins FliM, FliG, FliF, and PflI to the cell pole. (A) Schematic of the flagellum. The relative position of the MS ring (FliF), the switch complex (including FliG and FliM), the hook (FlgE), and the filament (flagellins) are indicated, as are the envelope layers: inner (cytoplasmic) membrane (IM), peptidoglycan layer (PG), outer membrane (OM), and $S$ layer (SL). (B) ECT imaging reveals densities corresponding to the intact flagellum, including the MS ring and the switch complex in or near the inner membrane of the wild type (WT) that are absent in the $\Delta t i p F$ mutant. The color-coding of the segmented structures corresponds to the color scheme in $A$. Flagellar structures (red) are shown in the left panel and in the segmentation of wild type. The black arrow points to the chemoreceptor array (orange) in $\Delta t i p F$ cells that is also present in wild type. Bars, $200 \mathrm{~nm} .(C)$ The subcellular localization of FliM-GFP from the $\mathrm{P}_{\text {van }}$ promoter on a medium copy number plasmid in a fliM mutant (fliM::Tn5), a tipF mutant ( $\Delta$ tipF; fliM:: Tn5), or a fliG mutant derivative ( $\Delta$ fliG; fliM::Tn5) analyzed by live-cell fluorescence microscopy. $(D)$ Localization of PflIYFP expressed from $\mathrm{P}_{p f I I}$ at the pfII locus in the presence $(p f l I-y f p)$ or absence of TipF $(\Delta t i p F ; p f l I-y f p)$ or TipN ( $\Delta t i p N ; p f l I-y f p)$. (DIC) Differential interference contrast images. $(E, F)$ Localization FliG-GFP $(E)$ and FliF-GFP $(F)$ expressed from $\mathrm{P}_{x y l}$ at the $x y l X$ locus in a $\triangle$ tipF mutant. 
dimensional (3D) tomograms to segmentation algorithms to reveal the densities of the flagellar MS ring and switch complex at the cytoplasmic membrane at the poles of wild-type cells but could not detect these structures at $\triangle$ tipF poles (Fig. 3A,B). The fact that chemoreceptor arrays that normally assemble at the same pole (Alley et al. 1992) and at the same time as the flagellum were seen in $\triangle t i p F$ cells confirms that the correct site was imaged (Fig. 3B, right panel).

Live-cell fluorescence imaging was used next to underscore the ECT results. To this end, we localized the FliM switch protein (Fig. 3C) as a C-terminal protein fusion to GFP (FliM-GFP) expressed from $\mathrm{P}_{\text {van }}$ in fliM::Tn5 cells harboring or not harboring a $\Delta$ fliG or $\Delta t i p F$ in-frame deletion. FliM is predicted to interact with the FliG switch protein that is tethered to the inner membrane via the FliF MS ring protein (Fig. 3A; Chevance and Hughes 2008). Consistent with this notion, FliM-GFP is diffuse in the cytoplasm of $\Delta f l i G$; fliM::Tn5 cells but is sequestered to the flagellated pole (opposite the stalk) in fliM::Tn5 cells (Fig. 3C). Moreover, FliM-GFP is delocalized in $\Delta t i p F$; fliM::Tn5 cells (Fig. 3C). Consistent with the dependence of polar localization of FliM on FliG, we confirmed that a derivative of the FliG switch protein fused C-terminally to GFP (FliG-GFP) also depends on TipF for polar localization (Fig. 3E; Supplemental Fig. S2C).

Since two switch proteins (FliG and FliM) are no longer polarized in the absence of TipF, we asked whether the MS ring protein FliF localizes in a TipF-dependent fashion using a partially functional FliF-GFP fusion expressed in the presence of endogenous FliF from the xylose-inducible promoter at the chromosomal xylX locus. Akin to FliGGFP, only few polar FliF-GFP foci were seen in $\triangle$ tipF cells (Fig. 3F; Supplemental Fig. S2B), providing further evidence that neither the MS ring nor the switch complex assembles in $\triangle t i p F$ cells. Interestingly, we found that FliFGFP required FliG to be sequestered to the pole (Supplemental Fig. S2D), suggesting that the mislocalization of FliF-GFP seen in $\triangle t i p F$ cells is a secondary effect of FliG dispersion. In support of this notion, we identified FliG as an interaction partner of soluble TipF in a yeast twohybrid assay, suggesting that TipF and FliG interact (see below).

Based on the result that FliF and FliG are dispensable for polar localization of TipF (data not shown), we conclude that TipF acts at the earliest known (nucleation) step in flagellum biogenesis, switch, and MS ring formation, respectively.

\section{The PflI flagellar positioning factor is recruited into a polar complex by TipF}

PflI is required for proper positioning of the flagellum and is recruited to the future flagellum assembly site before flagellar structural proteins are expressed by an unknown mechanism (Obuchowski and Jacobs-Wagner 2008). Like TipF, PflI is still polar in the absence of the MS ring protein FliF, raising the possibility that PflI localization is dependent on TipF. In support of this, we found TipF-mCherry to colocalize with PflI-GFP (PflI fused C-terminally to GFP) (Supplemental Fig. S3C) and observed that PfII-YFP or PflI-GFP are delocalized in $\triangle t i p F$ cells (Fig. $3 \mathrm{D}$; Supplemental Fig. S3A, respectively). Conversely, PflI does not noticeably affect TipF localization, as indicated by the apparent normal localization of TipF-GFP (expressed in lieu of endogenous TipF from the tipF locus) in $\Delta p f l I$ compared with wild-type cells (Supplemental Fig. S3B).

Since TipF is recruited to this site by the TipN landmark protein (Supplemental Fig. S3B), we predicted that PflI localization should also be dependent on TipN. Indeed, PflI-YFP or PflI-GFP foci are mispositioned near or within the stalk in $\Delta t i p N$ cells, a pattern resembling the misplacement of TipF-GFP in the absence of TipN (Fig. 3D; Supplemental Fig. S3A,B). The formation of these (mislocalized) PflI foci in $\Delta$ tipN cells is still TipF-dependent, as indicated by the dispersion of PflI-GFP in the envelope of $\Delta t i p F ; \Delta t i p N$ cells (Supplemental Fig. S3A). A similar dispersion of PflI-GFP occurs when the C-terminal prolinerich domain of PflI is deleted (residues 93-142 or 142-194) (Fig. 1B; Supplemental Fig. S3D), suggesting that this domain responds to the presence of TipF. In contrast, the $\Delta p f I I$ mutation does not seem to change the mislocalization of TipF-GFP in $\Delta t i p N$ cells (Supplemental Fig. S3B). Thus, flagellar assembly and recruitment events at the newborn pole proceed in the order TipN $>$ TipF $>[(\mathrm{Pfll})(\mathrm{FliF} / \mathrm{G} / \mathrm{M})]$.

To test whether this localization dependency is reflected in physical interactions among the proteins, we conducted pull-down experiments from extracts derived from cells harboring an empty vector control or a plasmid expressing either a TipF or PflI derivative with a C-terminal TAP (tandem affinity purification) tag (see the Materials and Methods) from the $\mathrm{P}_{\text {van }}$ promoter. Owing to the instability of TipF (described below), we conducted the pull-down experiments in mutant backgrounds $(\Delta t i p N$ and $f 1 b D:: \operatorname{Tn} 5$, when appropriate) in which TipF abundance is increased (Fig. 4A). This likely results from a posttranscriptional mechanism, as tipF transcription is unchanged in a tipN and $f l b D$ mutant (Supplemental Fig. S4A). Immunoblotting of TipF-TAP and PflI-TAP pulldown samples with antibodies to PflI (Fig. 4B) or TipF (Fig. 4C) provided evidence that TipF and PflI interact (directly or indirectly), but the pull down is inefficient. In contrast, coimmunoprecipitation of PflI with TipFGFP using monoclonal antibodies to GFP was efficient (Supplemental Fig. S4B,C,D). Moreover, immunoblotting of the TipF-TAP (Fig. 4D) and PflI-TAP (Fig. 4E) pulldown samples with polyclonal antibodies to TipN provided evidence that TipN is in a complex with TipF and PflI.

Finally, using an unbiased approach for interaction partners of TipF, we conducted a yeast two-hybrid screen using soluble TipF (lacking transmembrane segments, residues 1-90) as bait to probe a prey library of Caulobacter genomic fragments. Two positive clones, each encoding Gal4-AD fusions to a C-terminal fragment of FliG $\left(\mathrm{FliG}_{\mathrm{C}}\right)-$ residues 192-340 and residues 237-340 (Fig. 4F,G) —emerged from this screen. This result shows that FliG can directly interact with the soluble part of TipF and accounts for the localization dependency seen in vivo. 
A
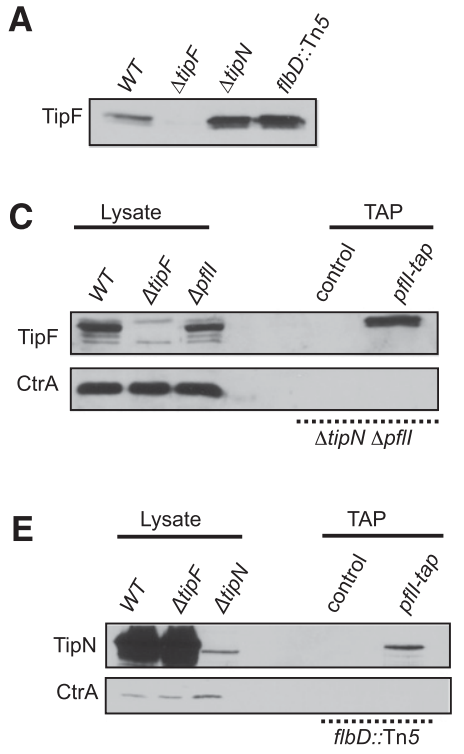

$\mathbf{F}$
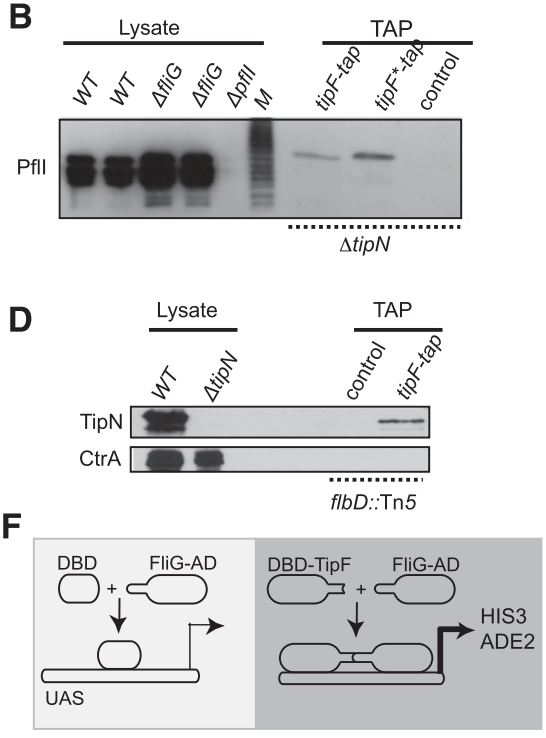

G

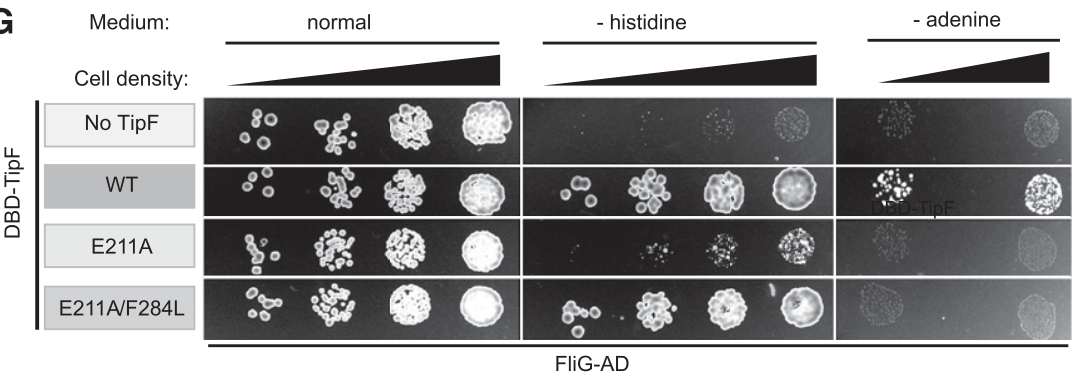

Figure 4. TipF directly interacts with FliG and forms a complex with TipN and PflI. $(A)$ TipF steady-state levels as determined by immunoblotting using polyclonal antibodies to TipF in lysates from wild-type, $\Delta t i p F$, $\Delta t i p N$, and flbD::Tn5. (B-E) TAP pull-down of TAP-tagged proteins expressed from $\mathrm{P}_{\text {van }}$ on a medium copy plasmid followed by immunoblotting using polyclonal antibodies to TipF, PflI, TipN, and CtrA. The cell lysates derived from boiled cells shown in the lanes at the left of the panels provide negative and positive controls for the specificity for the antisera. CtrA immunoblots are shown as a control for loading. (B) TipF-TAP and $\mathrm{TipF}^{\star}$-TAP / the E211A/F284L mutant is referred to as $\operatorname{TipF}^{\star}$ ) were expressed from the $\mathrm{P}_{\text {van }}$ promoter on a medium copy number plasmid in $\Delta$ tipN mutants. (M) Protein marker lane. $(C)$ TipF is present in PflI-TAP purifications of $\Delta t i p N \Delta p f I I$ double-mutant lysates. $(D, E)$ TipN is present in pull-downs of TipF-TAP $(D)$ or Pflli-TAP $(E)$ from $f 1 b D:$ : Tn5 lysates. $(F)$ Schematic showing the yeast two-hybrid $(\mathrm{Y} 2 \mathrm{H})$ assay using the $\mathrm{C}$ terminus of FliG (residues 237-340) as prey and the soluble part of TipF (residues 92-452) as bait to induce transcriptional activation of HIS3 and ADE2 (dark-gray box) as readout of the interaction. In the presence of DNA-binding domain (DBD)-TipF (TipF fused to the GAL4 $\mathrm{DBD})$, transcriptional activation is seen, but not when DBD is used without a TipF fusion (light-gray box). ( $G$ ) Growth of the yeast strains expressing the DBD-only (i.e., without TipF), wild-type, and mutant DBD-TipF variants along with FliG-AD (activation domain) on normal medium and selective medium lacking histidine (-histidine) or adenine (-adenine). The latter condition is more stringent. Gray scales from dark to light indicate the level of interaction corresponding to the growth readout on selective medium. Dark gray represents the strongest interaction as seen for wild-type DBD-TipF with FliG-AD.

Collectively, our results show that TipF interacts directly with FliG $_{\mathrm{C}}$ and assembles a flagellar organizational center comprising FliG, FliN, PflI, and TipN at the new pole.

\section{c-di-GMP induces TipF polarization and signaling}

If TipF is activated upon binding c-di-GMP, then cells expressing TipF variants that are unable to interact with c-di-GMP or cells depleted for c-di-GMP should also be unable to localize PflI. Neither the E211A derivative nor the D331A or K352A mutants were able to direct PflI into polar assemblies (Fig. 5A). Moreover, these inactive TipF mutants were themselves no longer sequestered to the pole (Fig. 5B). Furthermore, the $\operatorname{tipF}(E 211 \mathrm{~A})$ mutation phenocopies the flagellar assembly defect of the $\triangle t i p F$ deletion, as reflected in the absence of the FlgE hook protein (see Fig. 6F).

To test whether c-di-GMP depletion mimics the effects of the (c-di-GMP-binding defective) E211A mutant, we heterologously expressed the potent Pseudomonas aeruginosa PDE PA5295 in strains carrying the pflI-yfp reporter and imaged the resulting cells. Expression of PA5295, but not the catalytically inactive mutant PA5295-AAL [carrying an analogous mutation to $\operatorname{TipF}(\mathrm{E} 211 \mathrm{~A})$ at position E328], was previously shown to reduce c-di-GMP levels beyond detection (Duerig et al. 2009). We found that induction of
PA5295 (depletion of c-di-GMP) dispersed PflI-YFP from the pole (Fig. 6A). Consistent with these results, we observed that c-di-GMP depletion completely delocalized TipF-GFP from the pole (Fig. 6B), while TipN-GFP was still polar under these conditions (Fig. 6C). c-di-GMP depletion also prevented FlgE expression, akin to the $\triangle$ tipF or tipF(E211A) mutation (Fig. 6F). Interestingly, however, c-di-GMP depletion had a much stronger effect on transcription of the flgE gene than the $\triangle$ tipF or tipF(E211A) mutation (Fig. 6E), indicating that c-di-GMP modulates another step in flagellum biogenesis independently of TipF.

We conclude that c-di-GMP binding is a critical step in TipF activation and polarization. Interfering with this activating step by either introducing mutations in TipF that prevent c-di-GMP binding or depleting cells of c-diGMP locks TipF in a conformational state that prevents its activation and polarization, the recruitment of PflI, and flagellar assembly at the newborn pole.

\section{Bypassing the requirement of c-di-GMP for TipF signaling}

To illuminate how c-di-GMP activates and polarizes TipF, we isolated intragenic suppressor mutations that restore 
A

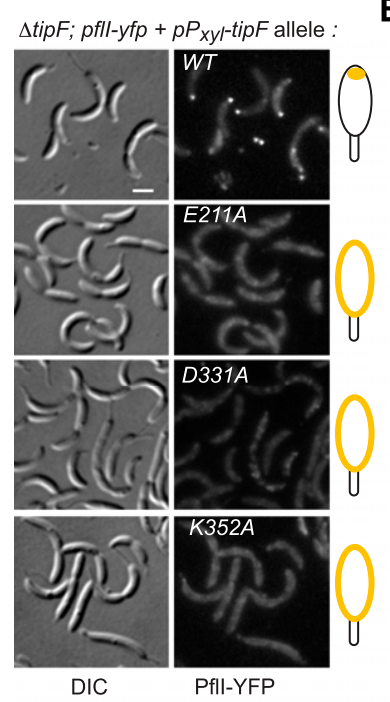

B

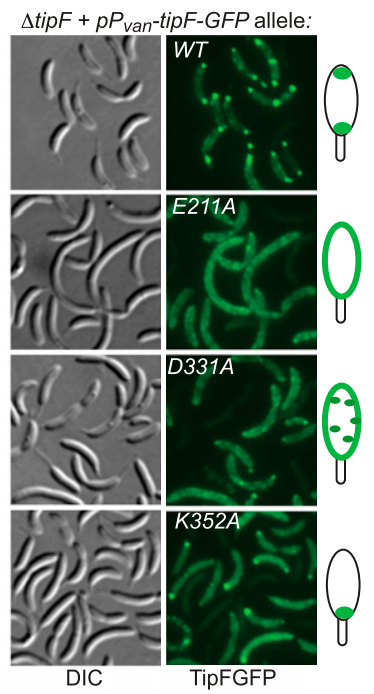

C

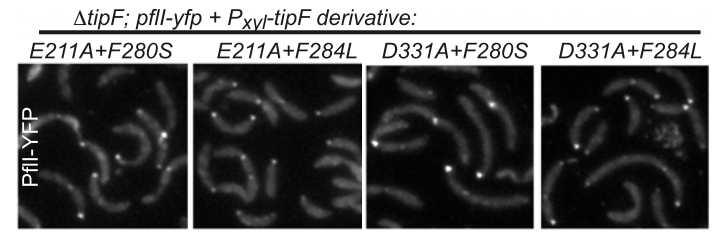

$\Delta$ DipF + $\mathrm{P}_{\text {van }}$-tipF-GFP derivative:

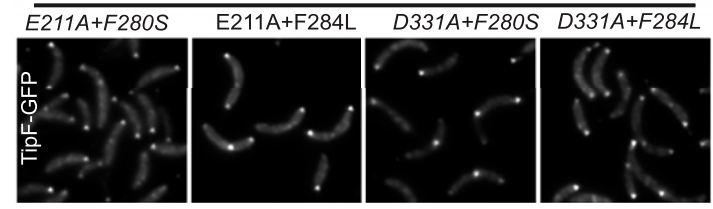

Figure 5. Suppressor mutations render TipF and PflI localization c-di-GMP-independent. $(A-D)$ Localization of PflI-YFP $(A, C)$ or TipF-GFP $(B, D)$ mutants in $\triangle t i p F$ cells harboring plasmids encoding TipF loss-of-function mutants expressed from the $\mathrm{P}_{x y l}(A, C)$ or the $\mathrm{P}_{\text {van }}(B, D)$ promoter a low copy number plasmid. (DIC) Differential interference contrast images. Note that $\operatorname{TipF}(\mathrm{K} 352 \mathrm{~A})$-GFP can cluster at the stalked pole but not at the newborn pole and that the ectopic signals at the stalked pole of wild-type (WT) TipF-GFP are due to (constitutive) expression of TipF from $\mathrm{P}_{\text {van }}$ on the lowcopy plasmid compared with constructs expressing TipF-GFP or YFP from the chromosome (cf. $B$ vs. Fig. 6B and Supplemental Fig. S3B).

motility to $\operatorname{tipF}(E 211 A)$ or $\operatorname{tipF}(D 331 A)$ cells. The suppressor alleles carry either a missense mutation in one of two proximal codons (F280S or F284L) within the c-diGMP-binding (EAL) domain or a duplication of a codon triplet (either ADA or DTV, residues 121-124 or 128-130, respectively) within the coiled-coil motif preceding the EAL domain (Fig. 1C,D). Importantly, the F280S or F284L missense mutations suppress either the tipF(E211A) or tipF(D331A) allele. As F280S or F284L are not allelespecific mutations, we considered the possibility that they also mitigate the localization defects of the D331A and E211A mutants. Indeed, C-terminal GFP fusions of the four double mutants (E211A/F280S, E211A/F284L, D331A/F280S, and D331A/F284L) revealed the proteins to be polarized and able to recruit PflI-YFP to the new pole (Fig. 5C,D).

If the suppressor mutations lock TipF in an activated state, then they might allow TipF to remain polarized under conditions of c-di-GMP depletion. We tested this idea by localizing YFP-tagged derivatives of wild-type, E211A/ F280S, and E211A/F284L versions of TipF expressed in $\Delta t i p F$ cells. While all three fusion proteins were polarized in the presence of c-di-GMP (Supplemental Fig. S5A), depletion of c-di-GMP dispersed wild-type TipF-YFP, but polar foci of the E211A/F280S and E211A/F284L versions persisted (Fig. 6D). Biochemical and biophysical analyses showed that the E211A/F284L double mutant is still unable to bind or hydrolyze c-di-GMP in vitro (Fig. 2A,B) and that the secondary structure is preserved (Supplemental Fig. S1). Interaction studies of wild-type and mutant TipF by yeast two-hybrid (Fig. 4G) and coimmunoprecipitation (Supplemental Fig. S4C,D) assays showed that $\operatorname{TipF}(\mathrm{E} 211 \mathrm{~A})$ associates poorly with FliG and PflI, but the suppressor mutations attenuated this effect of the E211A mutation.

To test whether these c-di-GMP bypass mutants can also support motility in the absence of c-di-GMP, we tested whether cells harboring suppressor mutations could swim in broth after depletion of c-di-GMP. As this was not the case (data not shown), we wondered whether c-di-GMP also promotes flagellar assembly or function at a later, TipF-independent step. We examined this possibility using the accumulation of the FlgE hook protein as a convenient proxy for defects in flagellar gene expression that can arise when assembly is blocked. Immunoblotting (Fig. 6F) revealed that FlgE steady-state levels (cell-associated and the hook accumulating in the supernatant) are reduced in c-di-GMP-depleted cells even in the presence of the E211A/F280S and E211A/F284L TipF versions. Transcription of flgE (probed using a $\mathrm{P}_{f l g E}-l a c Z$ transcriptional reporter) attained only $30 \% \pm 1 \%$ (E211A/F280S) and $30 \% \pm 1 \%(\mathrm{E} 211 \mathrm{~A} / \mathrm{F} 284 \mathrm{~L})$ of wild-type activity and was much below the activity level observed for the $\Delta t i p F$ single mutant $(57 \% \pm 1 \%$ of wild type) (Fig. $6 E)$. Moreover, $\mathrm{P}_{f l g E}-l a c Z$ reporter activity is further reduced (to $21 \%$ of wild type) when c-di-GMP is depleted in $\triangle t i p F$ cells, indicating that this c-di-GMP control step is required for efficient $f l g E$ transcription. A similar c-diGMP-dependent effect was seen on transcription of the fliL flagellin gene using the $\mathrm{P}_{f l i L}-1 a c Z$ transcriptional reporter (Supplemental Fig. S5B). In this case, however, c-di-GMP depletion reduces $f l j L$ transcription in $\Delta t i p F$ cells from near-full wild-type levels $(f l i L$ transcription is not affected by $\triangle t i p F$ mutation) to $32 \% \pm 2 \%$.

We conclude that TipF localization can be partially uncoupled from c-di-GMP control by the F280S and F284L suppressor mutations, but c-di-GMP influences at least one additional event in flagellum assembly, gene expression, localization, and/or (possibly affects) TipN polarization.

TipF abundance coincides with and depends on the cell cycle burst in c-di-GMP

Monitoring the steady-state levels of wild-type TipF and $\operatorname{TipF}(\mathrm{E} 211 \mathrm{~A})$ by immunoblotting suggested that (untagged) 
A

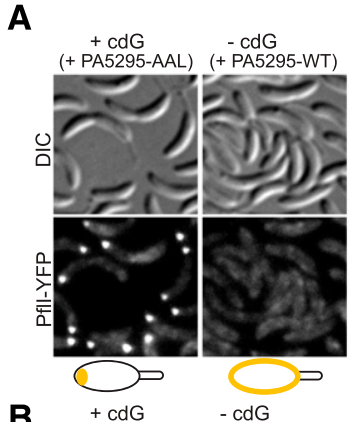

\section{B}

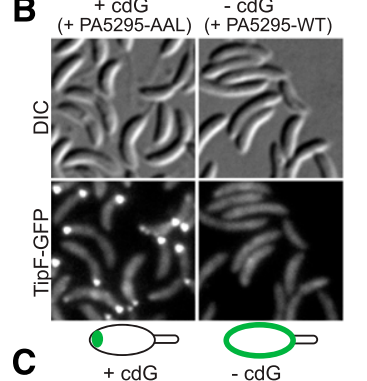

c

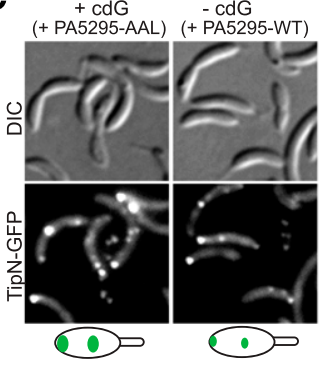

D

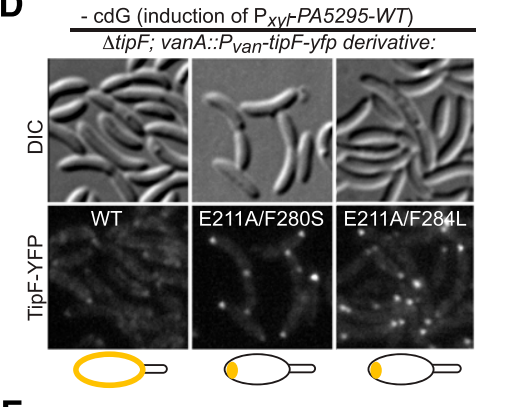

E

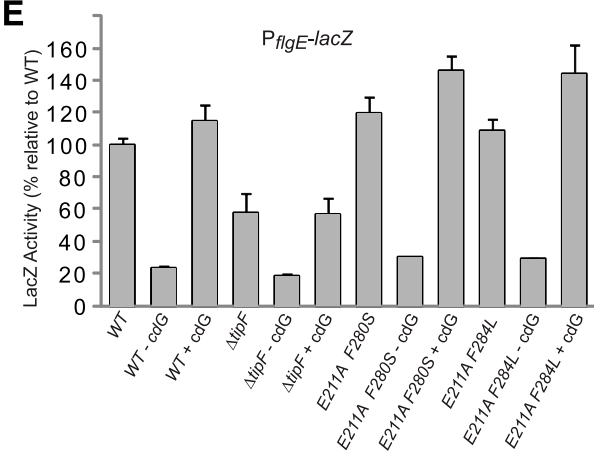

F ${ }_{\triangle t i p F}+p P_{\text {xyl-tipF }}$ derivative:

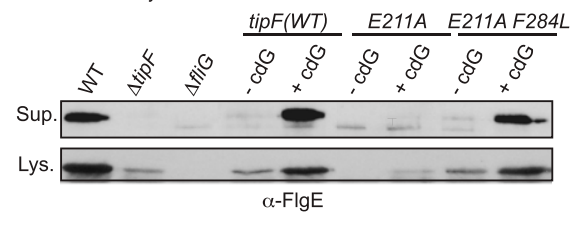

Figure 6. Localization of TipF and PflI as well as expression of FlgE is c-di-GMP-dependent. $(A-D)$ Localization of PflI-YFP $(A)$, TipF-GFP $(B)$, TipN-GFP $(C)$, and YFP-tagged TipF suppressor mutants $(D)$ under normal (PA5295-AAL, +cdG) or c-di-GMP-depleted (PA5295-WT, -cdG) conditions. PA5295 variants were expressed from the $\mathrm{P}_{x y l}$ promoter on a medium copy number plasmid in cells expressing the GFP fusion proteins from their respective promoters at the endogenous locus. In $D$, TipF-YFP variants were expressed from $\mathrm{P}_{\text {van }}$ at the vanA locus in $\Delta t i p F$ cells. (DIC) Differential interference contrast images. $(E)$ Effects of c-di-GMP concentration on transcription of the $f l g E$ hook gene by $\beta$-galactosidase assays of cells (wild-type [WT], $\Delta$ tipF, or $\Delta t i p F$ cells expressing mutant TipF from $\mathrm{P}_{\text {van }}$ at the vanA locus) harboring a $\mathrm{P}_{f l g E}-l a c Z$ transcriptional reporter fusion. $(F)$ Effects of c-di-GMP depletion on FlgE steady-state levels in supernatants (Sup.) or cell lysates (Lys.), determined by immunoblotting using anti-FlgE antibody ( $\alpha$-FlgE). TipF mutants were expressed from the $\mathrm{P}_{\mathrm{xyl}}$ promoter on a low copy number plasmid in $\Delta$ tipF cells.
TipF is stabilized upon binding c-di-GMP (Fig. 7A,B). The abundance of wild-type TipF expressed from $P_{x y l}$ (i.e., using the transcriptional and translational regulatory signals of the $x y l X$ gene) in $\triangle t i p F$ cells is significantly higher than that of the E211A version, suggesting that TipF is regulated at the level of stability by c-di-GMP. Indeed, half-life measurements of (untagged) wild-type TipF and TipF(E211A) under normal (Fig. 7B) or c-di-GMP-depleted (Fig. 7A) conditions (as described above) approximated the half-life of wild-type TipF at $>2 \mathrm{~h}$ in the presence of c-di-GMP and $<20 \mathrm{~min}$ in its absence. In contrast, for the E211A version, the half-life values were $\sim 10-20 \mathrm{~min}$ irrespective of the presence or absence of c-di-GMP, demonstrating that the E211A protein is not further destabilized upon c-di-GMP depletion (Fig. 7A,B). Remarkably, the half-life ( $\sim 40 \mathrm{~min})$ of the E211A/F284L variant was higher than that of the E211A single mutant but lower than that of wild-type TipF. Again, the half-life of this mutant was largely insensitive to the presence or absence of c-di-GMP, consistent with the persistence of E211A/ F284L at the pole in the absence of c-di-GMP (see above). Thus, when c-di-GMP is depleted, TipF is unable to adopt an active conformation and is destabilized. These events can be uncoupled from c-di-GMP with a mutation of F284L, which not only activates the E211A mutant form constitutively, but, at the same time, partially protects it from proteolysis in vivo.
In the search for a candidate protease that destabilizes TipF in vivo, we tested strains with mutations in all ATPdependent proteases and found that ClpX, the ATPdependent chaperone component of the ClpXP protease, controls the cell cycle-dependent accumulation of endogenous (untagged) TipF expressed from its native chromosomal location (Fig. 7C). Immunoblotting revealed TipF to be absent from G1-phase cells, induced during the $\mathrm{G} 1 \rightarrow \mathrm{S}$ transition $(40 \mathrm{~min})$, and diminished at the time of division (Fig. 7D). Poisoning ClpXP activity by expression of a dominant-negative (catalytically inactive) ClpX variant $\left(\mathrm{ClpX}^{\star}\right)$ (Osteras et al. 1999) from $\mathrm{P}_{\mathrm{xyl}}$, allows TipF accumulation in G1 cells (Fig. 7C), presumably because the normal turnover of TipF in G1 phase is blocked. Since a precipitous drop in c-di-GMP levels occurs in G1 cells (Paul et al. 2008; Christen et al. 2010) and TipF stability is clearly influenced by c-di-GMP abundance/binding, our results are consistent with the notion that the trough in c-di-GMP facilitates the turnover and thus the removal of TipF-dependent polarization during the cell cycle. In support of this view, fusion of GFP to the C terminus of TipF causes the aberrant accumulation of TipF in G1 phase (Fig. 7D) and allows TipF steady-state levels in unsynchronized populations to be maintained in the absence of c-di-GMP (Supplemental Fig. S6A,B). Similarly, comparison of the steady-state levels between untagged wild-type and TipF(E211A/F280S) expressed from 
A

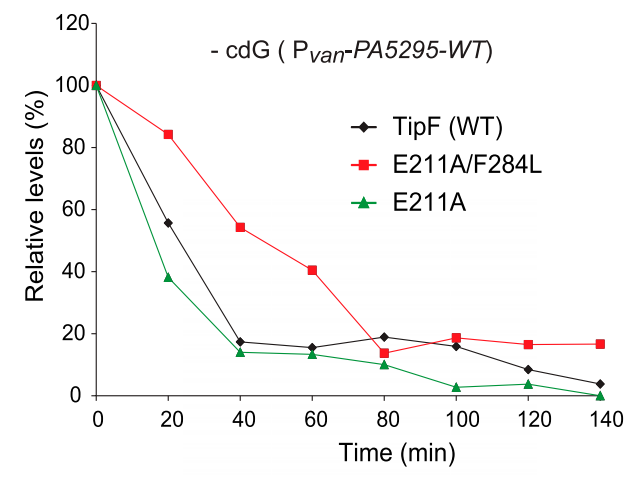

B

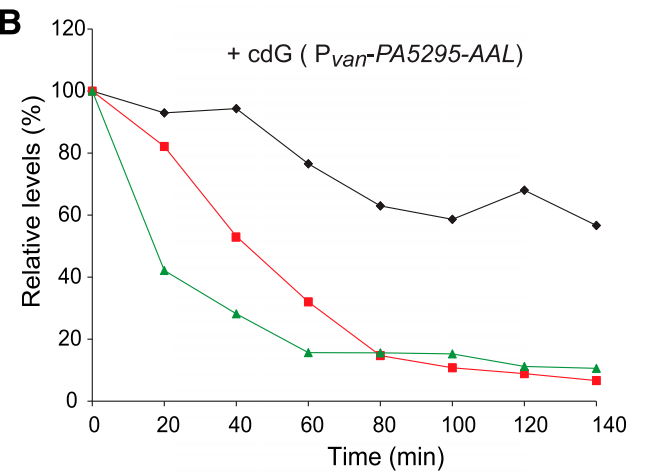

C

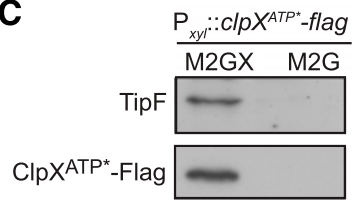

D

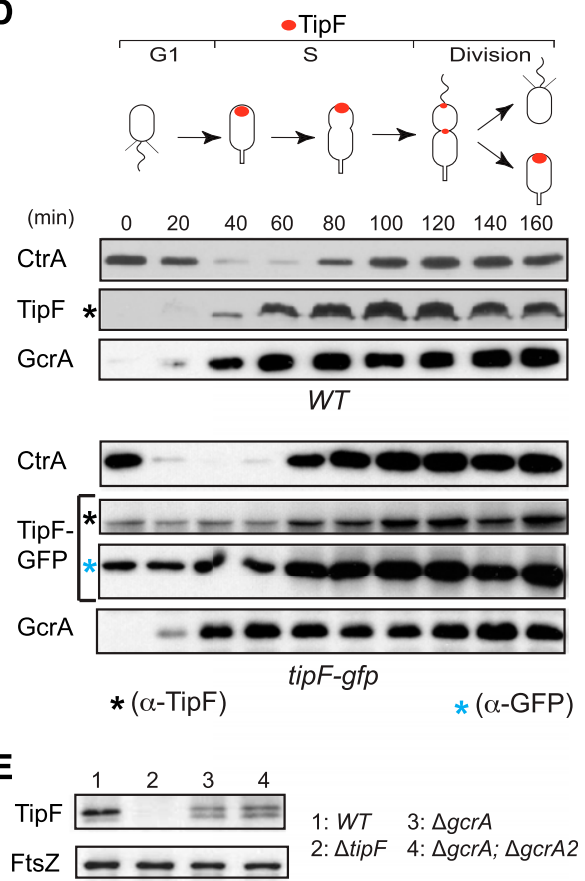

F GcrA bound to ( $\alpha$-GcrAqChIP) :
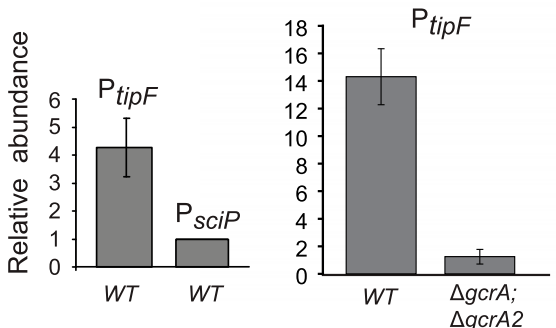

Figure 7. c-di-GMP levels affect TipF protein stability. $(A, B)$ Depletion of c-di-GMP by overexpression of a potent PDE reduces TipF steady-state levels. TipF, the c-di-GMP-binding mutant E211A, or the intragenic suppressor mutant E211A/F284L was expressed from $\mathrm{P}_{x y l}$ in $\Delta$ tipF cells grown in M2G containing xylose following a shift to M2G containing vanillate to induce the expression of the PDE PA5295 from $P$. aeruginosa or its active site mutant, PA5295-AAL, and repress $\mathrm{P}_{\mathrm{xy}}$. Samples were taken every 20 min, and protein levels were quantified from the immunoblots and plotted as percentages of the highest value. $(C)$ The dominant-negative $c l p X_{\mathrm{ATP}}$ allele was expressed from plasmid in M2G containing xylose (M2GX) or repressed by growing the cells in M2G. Swarmer cells were isolated, and TipF was detected using a polyclonal anti-TipF antibody and $\mathrm{ClpX}^{\mathrm{ATP}^{\star}}::$ Flag using monoclonal anti-M2 antibodies. In the presence of wild-type (WT) ClpXP, TipF was not detectable, while inactivation of ClpXP by the dominant-negative allele encoding ClpX ${ }^{\mathrm{ATP}}{ }^{\star}$ led to stabilization of TipF. $(D)$ The cell cycle abundance of TipF resembles that of GcrA. Synchronized wild-type or tipF-gfp (in which endogenous tipF is replaced by tipF-gfp) swarmer cells were released in fresh medium, and CtrA, TipF, TipF-GFP, and/or GcrA steadystate levels were determined by immunoblotting using antibodies to TipF (black asterisk), GFP (blue asterisk), CtrA, or GcrA at different times during cell cycle progression. $(E)$ TipF and FtsZ (control) steady-state levels in wild-type and mutant cells, as determined by immunoblotting using antibodies to TipF and FtsZ. $(F)$ GcrA binds the tipF promoter, as determined by qChIP experiments using polyclonal antibodies to GcrA. The abundance of the tipF and sciP promoters was quantified in the immunoprecipitates (left panel) and tipF (right panel) in wild-type and $\Delta g c r A ; \Delta g c r A 2$ double-mutant cells.

the xylose-inducible $\mathrm{P}_{x y l}$ promoter on a low-copy plasmid revealed that the stabilized E211A/F280S variant is more abundant than wild-type TipF in G1-phase cells (Supplemental Fig. S6C). Together, these findings support the model that the fluctuation of c-di-GMP during the cell cycle (i.e., its concentration trough in G1 cells) helps restrict TipF abundance to $S$ phase.

Additionally, the cell cycle abundance pattern of TipF is reinforced by timely synthesis, as is evident from the immunoblotting experiments in Figure 7D showing the increase of TipF during the cell cycle. Strikingly, the cell cycle pattern of TipF mirrors that of GcrA, a master transcriptional regulator that is required for the accumulation of a myriad of cell cycle-regulated transcripts, including $t i p F$, at the G1 $\rightarrow \mathrm{S}$ transition (Holtzendorff et al. 2004). Consistent with the hypothesis that GcrA directly activates transcription of the TipF-encoding gene at the G1 $\rightarrow$ S transition, we observed that GcrA is required for efficient TipF protein accumulation (Fig. 7E), and quantitative chromatin immunoprecipitation (qChIP) 
analysis using polyclonal antibodies to GcrA revealed that GcrA binds the tipF promoter, but not the sciP promoter, in vivo in a GcrA-dependent manner (Fig. 7F). The induction of TipF expression at the transcriptional level by GcrA and the concordant surge in c-di-GMP levels at the $\mathrm{G} 1 \rightarrow \mathrm{S}$ transition ensure that TipF is active and ready to prime flagellum biosynthesis at the newborn pole, while a drop in c-di-GMP in the incipient G1 daughter cells results in the removal of TipF, thus resetting the flagellar polarity cascade for the next cell cycle.

\section{Discussion}

The establishment, amplification, and propagation of polarity cues during the cell cycle are hallmarks of all polarized cells, from bacterial to metazoan origin. Here we elucidate a mechanism that not only implements but also serves to subsequently reset a polarity pathway during the cell cycle in response to cyclic nucleotide signaling. While nucleotide-based signals direct critical events in the establishment and/or reinforcement of polarity cues in eukaryotes (Slaughter et al. 2009; Charest and Firtel 2010; St Johnston and Ahringer 2010; Amato et al. 2011; Ji and Tulin 2012), the underlying mechanisms are not well understood in bacterial systems. Using polar flagellum morphogenesis as a proxy to unravel how and when polarity cues are instated by cyclic nucleotide signaling during the Caulobacter cell cycle, we found that the presence of c-di-GMP activates and amplifies the TipF-dependent flagellar polarization pathway, while its removal promotes the elimination of TipF from G1 cells and provides a (possible checkpoint-like) mechanism, allowing the re-establishment of the flagellar polarization pathway to be coordinated with other developmental events at the G1 $\rightarrow$ S transition.

At least four regulatory factors (TipN, GcrA, ClpX, and c-di-GMP) impart spatiotemporal control on TipF through interwoven mechanisms acting at different levels. c-diGMP adopts a central position in this regulatory web, implementing its effects by directly binding TipF (Fig. 2). The induction of TipF at the G1 $\rightarrow$ S transition is reinforced by two concurrent mechanisms: its synthesis triggered by the GcrA master regulator and its stabilization by c-di-GMP (Fig. 1A). Both mechanisms ensure that TipF accumulation is coordinated to other critical morphogenetic events that are activated at the same time. GcrA promotes the expression of genes encoding determinants for polarity (PodJ, controlling morphogenesis of pili), division (MipZ, a division inhibitor), and asymmetry (PleC, a histidine kinase/phosphatase) (Viollier et al. 2002; Holtzendorff et al. 2004; Thanbichler and Shapiro 2006). The concurrent accumulation of c-di-GMP protects TipF from ClpXP-dependent proteolysis and thus reinforces the surge of TipF while also promoting the degradation of CtrA at the G1 $\rightarrow$ S transition via the PopA effector and stalk biogenesis by an unknown mechanism (Paul et al. 2004, 2008; Duerig et al. 2009; Abel et al. 2011). Importantly, c-di-GMP binding is required not only to activate and polarize TipF, but also for the TipF-dependent recruitment of flagellar assembly factors and regulators such as PflI, FliG, and FliM to the new pole. The underlying conformational rearrangements that likely occur when TipF binds c-di-GMP can be blocked by mutational destruction of the c-di-GMP-binding site or when c-diGMP is depleted from cells. While these manipulations abrogate TipF polarization and function, we isolated compensatory missense mutations in the c-di-GMP-binding domain that reverse these effects and also allow TipF localization under conditions of c-di-GMP depletion. The same motility suppressor screen was answered by triplet codon duplications in a predicted coiled-coilrich region that precedes the c-di-GMP-binding domain (Fig. 1B). Helical wheel analysis of these mutant proteins predicts that the $\alpha$-helicity is maintained by the duplication while causing a shift of the hydrophobic region that could cause structural rearrangements. This rearrangement likely also underlies the interaction of TipF•c-di-GMP with TipN. During the late stages of constriction of the preceding cell cycle, the TipN landmark signal is deposited at the newborn pole to ensure correct interpretation of the polarity axis in the progeny (Huitema et al. 2006; Lam et al. 2006). Because of this spatial cue, the subsequent events triggered by c-diGMP at the G1 $\rightarrow$ S transition are executed at the correct subcellular site (i.e., the newborn pole) (Fig. 1A). In support of this, our pull-down experiments revealed TipN to be part of this flagellar organizational complex that includes TipF, Pfl, and at least one flagellar switch component (Fig. 2A).

The flagellar switch complex is best known as a target to regulate flagellar rotation but fulfills a fundamental role in flagellar assembly (Irikura et al. 1993; Chevance and Hughes 2008; Davis and Viollier 2011). Indeed the FliG switch protein interacts with the FliF MS ring protein and the MotA stator protein along with other switch proteins. This central position of FliG in flagellar assembly and function has been exploited to regulate motility in response to specific developmental states or nutritional and systemic cues (Ryjenkov et al. 2006; Blair et al. 2008; Paul et al. 2011; Zarbiv et al. 2012). Despite the dual role of FliG, no mechanism has been described to regulate flagellar assembly through FliG. TipF is the first representative of this class, as the soluble portion of TipF (encompassing the predicted coiled-coil and EAL domains) interacts directly with the C-terminal portion $\left(\right.$ FliG $\left._{\mathrm{C}}\right)$. The role of c-di-GMP in flagellar assembly likely goes beyond the TipF-FliG interaction, as indicated by the existence of an apparent TipF-independent, but c-diGMP-dependent, flagellar assembly step (Fig. 6E,F).

The FlhF GTPase of vibrios, pseudomonads, and Campylobacter jejuni may function akin to the TipF and c-di-GMP-dependent flagellar polarization pathway (Pandza et al. 2000; Salvetti et al. 2007; Balaban et al. 2009; Kusumoto et al. 2009). FlhF is polarized and recruits the MS ring protein FliF to the polar site of flagellation in Vibrio. cholerae (Green et al. 2009). However, despite the pervasive polar flagellation found across bacterial lineages, FlhF polarization, activation, and function are poorly understood. Interestingly, the Myxococcus xanthus Ras-like GTPases MglA and SofG are used to direct motility pro- 
teins required for flagellum-independent (gliding) motility to the pole (Leonardy et al. 2010; Zhang et al. 2010; Bulyha et al. 2013). Moreover, a member of the ParA/MinD family of ATPases positions the chemosensory apparatus at the pole in V. cholera (Ringgaard et al. 2011; Yamaichi et al. 2012), and a related protein ensures the medial placement of the chemosensory apparatus in Rhodobacter sphaeroides (Thompson et al. 2006), but the temporal relationships with the cell cycle are unexplored in these systems.

c-di-GMP-mediated spatiotemporal control of polar morphogenesis extends far beyond the $\alpha$-proteobacterial lineage. In the $\gamma$-proteobacterium $P$. aeruginosa, an imbalance in c-di-GMP abundance is observed in the two progeny compartments of a dividing cell, with the trough in c-di-GMP levels occurring in the daughter compartment bearing the polar flagellum (Christen et al. 2010). FimX, an EAL-GGDEF domain hybrid protein from $P$. aeruginosa, is polarly localized and promotes the assembly of retracting type IV (polar) pili (Tfp) for twitching motility. Importantly, FimX influences the positioning of Tfp and uses an EAL domain to bind c-di-GMP and induce (long-range) conformational change in the adjacent domain (Huang et al. 2003). However, it is unknown how FimX scouts the pole, how it signals Tfp assembly, and how its localization is coordinated with cell cycle progression. We show that spatiotemporal cues from pre-existing spatial landmarks, the c-di-GMP second messenger, and the cell cycle transcriptional program are integrated at TipF to orchestrate the periodic removal and re-establishment of a polarity pathway controlling morphogenesis during the cell cycle. Thus, small nucleotide-induced polarization pulses that drive morphogenesis at key times in the cell cycle appear to have been invented more than once during evolution from prokaryotic to metazoan cells and may thus even function akin in eukaryotic organelles.

\section{Materials and methods}

\section{Coimmunoprecipitation}

Cells $(50 \mathrm{~mL})$ were grown to mid-log phase in the presence of $50 \mathrm{mM}$ vanillate inducer, harvested, washed three times in buffer I $(50 \mathrm{mM}$ sodium phosphate at $\mathrm{pH} 7.4,50 \mathrm{mM} \mathrm{NaCl}, 1 \mathrm{mM}$ EDTA), and resuspended in buffer II (50 $\mathrm{mM}$ sodium phosphate at $\mathrm{pH} 7.4,50 \mathrm{mM} \mathrm{NaCl}, 0.5 \%$ n-dodecyl- $\beta$-D-maltoside, two protease inhibitor tablets [Complete EDTA-free, Roche]) containing $1 \times$ Ready-Lyse lysozyme solution (Epicentre), $2 \mathrm{mM} \mathrm{MgCl}_{2}, 1$ $\mathrm{mM}$ EDTA, and $30 \mathrm{U}$ of DNase I (Roche). The solution was incubated for $20 \mathrm{~min}$ at room temperature and subsequently centrifuged at $10,000 \mathrm{~g}$ for $3 \mathrm{~min}$ at $4^{\circ} \mathrm{C}$ to remove cellular debris. The supernatant was precleared with $50 \mu \mathrm{L}$ of Proteinase-G agarose beads (Roche). To the precleared solution, mouse monoclonal anti-GFP antibodies (1:300 dilution; Living Colors A.V. monoclonal antibody [JL-8], Clontech, I was added and incubated for $90 \mathrm{~min}$ at $4^{\circ} \mathrm{C}$. The antibody-protein complexes were trapped with Proteinase-G agarose beads washed once with buffer I containing $0.5 \% \mathrm{n}$-dodecyl- $\beta$-D-maltoside, twice with $1 \%$ immunoprecipitation buffer (Protein G immunoprecipitation kit, SigmaAldrich), and once with $0.1 \%$ immunoprecipitation buffer to remove salts and finally resuspended in $70 \mu \mathrm{L}$ of $1 \times$ Laemmli sample buffer. After boiling and centrifugation, precipitated pro- teins were identified by immunoblotting using polyclonal antibodies to PflI or CtrA.

\section{TAP}

The TAP procedure was based on that described by Rigaut et al. (1999). Briefly, cells (1 L) were grown to mid-log phase in the presence of $50 \mathrm{mM}$ vanillate for $3 \mathrm{~h}$ and harvested by centrifugation at $6000 \mathrm{~g}$ for $10 \mathrm{~min}$. The cells were then washed in buffer I ( $50 \mathrm{mM}$ sodium phosphate at $\mathrm{pH} 7.4,50 \mathrm{mM} \mathrm{NaCl}, 1 \mathrm{mM}$ EDTA) and lysed for $15 \mathrm{~min}$ at room temperature in $500 \mu \mathrm{L}$ of buffer II (buffer I + 10\% n-dodecyl- $\beta$-D-maltoside, two protease inhibitor tablets [Complete EDTA-free, Roche], $1 \times$ Ready-Lyse lysozyme [Epicentre], $500 \mathrm{U}$ of DNase I [Roche], $2 \mathrm{mM} \mathrm{MgCl}, 1 \mathrm{mM}$ EDTA). The volume of the solution was brought up to $10 \mathrm{~mL}$ with buffer I and incubated for $15 \mathrm{~min}$ at room temperature. Cellular debris was removed by centrifugation at $7000 \mathrm{~g}$ for $15 \mathrm{~min}$ at $4^{\circ} \mathrm{C}$, and the salt concentration of the supernatant was adjusted by adding $10 \mu \mathrm{L}$ of $1 \mathrm{M}$ Tris per milliliter sample, $20 \mu \mathrm{L}$ of $5 \mathrm{M} \mathrm{NaCl}$ per milliliter sample, and $25 \mu \mathrm{L}$ of $10 \%$ n-dodecyl- $\beta$-D-maltoside per milliliter sample. The supernatant was incubated for $2 \mathrm{~h}$ at $4^{\circ} \mathrm{C}$ with IgG Sepharose beads (GE Healthcare Biosciences) that had been washed once with IPP150 buffer (10 mM Tris- $\mathrm{HCl}$ at $\mathrm{pH} 8,150 \mathrm{mM} \mathrm{NaCl}, 0.25 \%$ n-dodecyl$\beta$-D-maltoside). After incubation, the beads were washed at $4^{\circ} \mathrm{C}$ three times with IPP150 buffer and once with TEV cleavage buffer (10 mM Tris- $\mathrm{HCl}$ at $\mathrm{pH} \mathrm{8,150} \mathrm{mM} \mathrm{NaCl}, 0.25 \%$ n-dodecyl- $\beta$-Dmaltoside, $0.5 \mathrm{mM}$ EDTA, $1 \mathrm{mM}$ DTT). The beads were then incubated overnight at $4^{\circ} \mathrm{C}$ with $1 \mathrm{~mL}$ of TEV solution (TEV cleavage buffer with $100 \mathrm{U}$ of TEV protease per milliliter [Promega]) to release the tagged complex. $\mathrm{CaCl}_{2}(3 \mu \mathrm{M})$ was added to the solution and incubated for $1 \mathrm{~h}$ at $4^{\circ} \mathrm{C}$ with calmodulin beads (GE Healthcare Biosciences) that had been washed once with calmodulin-binding buffer $(10 \mathrm{mM} \beta$-mercaptoethanol, $10 \mathrm{mM}$ Tris- $\mathrm{HCl}$ at $\mathrm{pH} 8,150 \mathrm{mM} \mathrm{NaCl}, 1 \mathrm{mM}$ magnesium acetate, $1 \mathrm{mM}$ imidazole, $2 \mathrm{mM} \mathrm{CaCl}_{2}, 0.25 \%$ n-dodecyl- $\beta$-Dmaltoside). After incubation, the beads were washed three times with calmodulin-binding buffer and eluted with IPP150 calmodulin elution buffer (calmodulin-binding buffer substituted with 2 mM EGTA instead of $\mathrm{CaCl}_{2}$ ). The eluate was then concentrated with Biomax centrifugal filter tubes (Millipore).

\section{Protein overexpression and purification for PDE assay} and ITC analyses

E. coli BL21 cells carrying the respective wild-type and mutant TipF overexpression plasmids were grown in LB medium, and expression was induced by adding isopropyl 1 -thio- $\beta$-d-galactopyranoside at $\mathrm{A} 600=0.4$ to a final concentration of $1 \mu \mathrm{M}$. Cells were centrifuged and resuspended in buffer containing $20 \mathrm{mM}$ Tris- $\mathrm{HCl}$ (pH 7), $250 \mathrm{mM} \mathrm{NaCl}, 5 \mathrm{mM}$ imidazole, $10 \mathrm{mM} \mathrm{MgCl}_{2}, 1 \%$ glycerol, and Complete minicocktail of EDTA-free protease inhibitors at the concentrations specified by the manufacturer (Roche). Cells were lysed by passage through a French pressure cell, and the suspension was clarified by centrifugation at $15,000 \mathrm{~g}$ for $40 \mathrm{~min}$. The supernatant was incubated with pre-equilibrated Profinity IMAC Ni-resin (Bio-Rad) for $1 \mathrm{~h}$ at $4^{\circ} \mathrm{C}$. The resins were washed, and the proteins were eluted with $350 \mathrm{mM}$ imidazole. The eluate was loaded on a HiLoad 16/60 Superdex 75 prep-grade gel filtration column (GE Healthcare; FPLC system: AEKTA Purifier, Amersham Biosciences) for the next purification step using $50 \mathrm{mM}$ Tris- $\mathrm{HCl}(\mathrm{pH}$ 8) and $50 \mathrm{mM} \mathrm{NaCl}$ buffer. The monomer fractions were collected and concentrated (Amicon Ultra 10,000-MW centrifugal filters, Millipore) for further assays. The protein concentrations were determined using the Bradford assay (Bio-Rad). 
Davis et al.

PDE assay

Wild-type and mutant TipF were purified as His6-tagged variants using standard conditions (see the Supplemental Material) and tested for c-di-GMP PDE activity. As a control, PDE class A YahA from E. coli was used. For each protein, a $10 \mu \mathrm{M}$ final concentration was incubated with $100 \mu \mathrm{M}$ c-di-GMP (synthesized enzymatically and purified via FPLC reverse-phase chromatography) in $50 \mathrm{mM}$ Tris- $\mathrm{HCl}$ (pH 8), $50 \mathrm{mM} \mathrm{NaCl}, 5 \mathrm{mM} \mathrm{MgCl}_{2}$, and $1 \mathrm{mM}$ DTT at room temperature. Samples were taken at time points $0 \mathrm{~h}, 1 \mathrm{~h}, 2 \mathrm{~h}$, and overnight. The reactions were stopped by heating for $5 \mathrm{~min}$ at $99^{\circ} \mathrm{C}$, diluted with $5 \mathrm{mM} \mathrm{NH}_{4} \mathrm{HCO}_{3}(\mathrm{pH} 8)$, filtered $(0.22 \mu \mathrm{M})$, and analyzed on a Resource $\mathrm{Q}$ ion-exchange chromatography column (GE Healthcare).

ITC

The interaction of TipF with c-di-GMP was measured with a VP-ITC isothermal titration calorimeter from MicroCal, with $\operatorname{TipF}(32.5 \mu \mathrm{M})$ in the calorimeter cell and c-di-GMP $(118 \mu \mathrm{M})$ in the injection syringe (buffer: $50 \mathrm{mM}$ Tris/ $\mathrm{HCl}, 50 \mathrm{mM} \mathrm{NaCl}$ at $\mathrm{pH} 8$ at $25^{\circ} \mathrm{C}$. All solutions were degassed and equilibrated at the desired temperature $\left(25^{\circ} \mathrm{C}\right.$ or $\left.35^{\circ} \mathrm{C}\right)$ before using. The delay between the injections was set to $5 \mathrm{~min}$ to ensure re-equilibration between injections. Data were evaluated using Origin software (OriginLab).

\section{Cryoelectron microscopy sample preparation}

C. crescentus grown in PYE medium were flash-frozen (plungefrozen) onto glow-discharged, 200 mesh, Quantifoil copper grids (Quantifoil) in liquid ethane using a Vitrobot Mark II system (FEI). BSA-treated colloidal gold $(10 \mathrm{~nm})$ was applied to the grids and air-dried before sample application and plunge-freezing.

\section{Cryoelectron microscopy data collection}

Cryoelectron tomography data collection was performed with an FEI Tecnai G ${ }^{2}$ F30 at the University of Minnesota Characterization Facility. Images were acquired with a Gatan $4 \mathrm{k} \times 4 \mathrm{k}$ Ultrascan charged-couple device (CCD camera) with a pixel size of $0.372 \mathrm{~nm}($ at $31,000 \times$ ) or $0.495 \mathrm{~nm}$ (at $23,000 \times$ ) on the specimen. A total electron dose of 150 electrons per squared angstrom $\left(\mathrm{e}-/ \AA^{2}\right)$ was used to collect a full-tilt series ranging from $-65^{\circ}$ to $+65^{\circ}$ (131 images). Data were collected at a defocus of $8.0 \mu \mathrm{m}$ under focus (first CTF 0: $1 / 4 \mathrm{~nm}^{-1}$ ). Images were acquired automatically with $1^{\circ}$ tilt steps using the predictive University of California at San Francisco tomography package (Zheng et al. 2007).

\section{Cryoelectron image processing and analysis}

Tomographic reconstructions were generated using IMOD (Kremer et al. 1996). The Amira visualization software package (Visage Imaging Systems, Inc.) was used for volume rendering and material labeling (segmentation). A visualization and segmentation toolbox (Pruggnaller et al. 2008) developed specifically for $3 \mathrm{D}$ electron microscopy data analysis in Amira was used to achieve a more objective segmentation of the data by avoiding manual contouring (entirely subjective tracing of edges or features). After contrast inversion, slices were filtered using a 3D Gaussian smoothing with a kernel size of $3 \times 3 \times 3$. Subsequently, the IslandLabel module was applied to binarize the data with a threshold and tag connected regions of the binary image with a label.
Construction of strains and plasmids, immunoblotting, yeast two-hybrid analyses, and fluorescence microscopy

Descriptions of the construction of strains and plasmids, immunoblotting, yeast two-hybrid analyses, and fluorescence microscopy can be found in the Supplemental Material.

\section{Acknowledgments}

We thank Lucy Shapiro and Jim Gober for providing antibodies. Funding support is from Swiss National Science Foundation (no. 127287 to P.H.V. and no. 130469 to U.J.), National Institutes of Health (GM104540 to E.R.W), and Human Frontier Science Program (RGP0051/2010 to P.H.V and E.R.W).

\section{References}

Abel S, Chien P, Wassmann P, Schirmer T, Kaever V, Laub MT, Baker TA, Jenal U. 2011. Regulatory cohesion of cell cycle and cell differentiation through interlinked phosphorylation and second messenger networks. Mol Cell 43: 550560.

Alley MR, Maddock JR, Shapiro L. 1992. Polar localization of a bacterial chemoreceptor. Genes Dev 6: 825-836.

Amato S, Liu X, Zheng B, Cantley L, Rakic P, Man HY. 2011. AMP-activated protein kinase regulates neuronal polarization by interfering with PI 3-kinase localization. Science 332: 247-251.

Balaban M, Joslin SN, Hendrixson DR. 2009. FlhF and its GTPase activity are required for distinct processes in flagellar gene regulation and biosynthesis in Campylobacter jejuni. J Bacteriol 191: 6602-6611.

Barends TR, Hartmann E, Griese JJ, Beitlich T, Kirienko NV, Ryjenkov DA, Reinstein J, Shoeman RL, Gomelsky M, Schlichting I. 2009. Structure and mechanism of a bacterial light-regulated cyclic nucleotide phosphodiesterase. Nature 459: 1015-1018.

Blair KM, Turner L, Winkelman JT, Berg HC, Kearns DB. 2008. A molecular clutch disables flagella in the Bacillus subtilis biofilm. Science 320: 1636-1638.

Boehm A, Kaiser M, Li H, Spangler C, Kasper CA, Ackermann M, Kaever V, Sourjik V, Roth V, Jenal U. 2010. Second messenger-mediated adjustment of bacterial swimming velocity. Cell 141: 107-116.

Boyd CD, O'Toole GA. 2012. Second messenger regulation of biofilm formation: Breakthroughs in understanding c-diGMP effector systems. Annu Rev Cell Dev Biol 28: 439462.

Brown MT, Delalez NJ, Armitage JP. 2011. Protein dynamics and mechanisms controlling the rotational behaviour of the bacterial flagellar motor. Curr Opin Microbiol 14: 734740.

Bulyha I, Lindow S, Lin L, Bolte K, Wuichet K, Kahnt J, van der Does C, Thanbichler M, Sogaard-Andersen L. 2013. Two small GTPases act in concert with the bactofilin cytoskeleton to regulate dynamic bacterial cell polarity. Dev Cell 25: 119131.

Charest PG, Firtel RA. 2010. 'TORCing' neutrophil chemotaxis. Dev Cell 19: 795-796.

Chevance FF, Hughes KT. 2008. Coordinating assembly of a bacterial macromolecular machine. Nat Rev Microbiol 6: 455-465.

Christen M, Kulasekara HD, Christen B, Kulasekara BR, Hoffman LR, Miller SI. 2010. Asymmetrical distribution of the second messenger c-di-GMP upon bacterial cell division. Science 328: $1295-1297$. 
Davis NJ, Viollier PH. 2011. Probing flagellar promoter occupancy in wild-type and mutant Caulobacter crescentus by chromatin immunoprecipitation. FEMS Microbiol Lett 319: 146-152.

Duerig A, Abel S, Folcher M, Nicollier M, Schwede T, Amiot N, Giese B, Jenal U. 2009. Second messenger-mediated spatiotemporal control of protein degradation regulates bacterial cell cycle progression. Genes Dev 23: 93-104.

Fang X, Gomelsky M. 2010. A post-translational, c-di-GMPdependent mechanism regulating flagellar motility. Mol Microbiol 76: 1295-1305.

Green JC, Kahramanoglou C, Rahman A, Pender AM, Charbonnel N, Fraser GM. 2009. Recruitment of the earliest component of the bacterial flagellum to the old cell division pole by a membrane-associated signal recognition particle family GTP-binding protein. J Mol Biol 391: 679-690.

Hengge R. 2009. Principles of c-di-GMP signalling in bacteria. Nat Rev Microbiol 7: 263-273.

Holtzendorff J, Hung D, Brende P, Reisenauer A, Viollier PH, McAdams HH, Shapiro L. 2004. Oscillating global regulators control the genetic circuit driving a bacterial cell cycle. Science 304: 983-987.

Huang B, Whitchurch CB, Mattick JS. 2003. FimX, a multidomain protein connecting environmental signals to twitching motility in Pseudomonas aeruginosa. I Bacteriol 185: 7068-7076.

Huitema E, Pritchard S, Matteson D, Radhakrishnan SK, Viollier PH. 2006. Bacterial birth scar proteins mark future flagellum assembly site. Cell 124: 1025-1037.

Irikura VM, Kihara M, Yamaguchi S, Sockett H, Macnab RM. 1993. Salmonella typhimurium fliG and fliN mutations causing defects in assembly, rotation, and switching of the flagellar motor. J Bacteriol 175: 802-810.

Ji Y, Tulin AV. 2012. Poly(ADP-ribose) controls DE-cadherindependent stem cell maintenance and oocyte localization. Nat Commun 3: 760.

Kremer JR, Mastronarde DN, McIntosh JR. 1996. Computer visualization of three-dimensional image data using IMOD. I Struct Biol 116: 71-76.

Kusumoto A, Nishioka N, Kojima S, Homma M. 2009. Mutational analysis of the GTP-binding motif of FlhF which regulates the number and placement of the polar flagellum in Vibrio alginolyticus. I Biochem 146: 643650.

Lam H, Schofield WB, Jacobs-Wagner C. 2006. A landmark protein essential for establishing and perpetuating the polarity of a bacterial cell. Cell 124: 1011-1023.

Laub MT, McAdams HH, Feldblyum T, Fraser CM, Shapiro L. 2000. Global analysis of the genetic network controlling a bacterial cell cycle. Science 290: 2144-2148.

Leonardy S, Miertzschke M, Bulyha I, Sperling E, Wittinghofer A, Sogaard-Andersen L. 2010. Regulation of dynamic polarity switching in bacteria by a Ras-like G-protein and its cognate GAP. $E M B O$ J 29: 2276-2289.

McAdams HH, Shapiro L. 2011. The architecture and conservation pattern of whole-cell control circuitry. J Mol Biol 409: 28-35.

Obuchowski PL, Jacobs-Wagner C. 2008. PflI, a protein involved in flagellar positioning in Caulobacter crescentus. J Bacteriol 190: $1718-1729$.

Osteras M, Stotz A, Schmid Nuoffer S, Jenal U. 1999. Identification and transcriptional control of the genes encoding the Caulobacter crescentus ClpXP protease. I Bacteriol 181: 3039-3050.

Pandza S, Baetens M, Park CH, Au T, Keyhan M, Matin A. 2000. The G-protein FlhF has a role in polar flagellar placement and general stress response induction in Pseudomonas putida. Mol Microbiol 36: 414-423.

Paul R, Weiser S, Amiot NC, Chan C, Schirmer T, Giese B, Jenal U. 2004. Cell cycle-dependent dynamic localization of a bacterial response regulator with a novel di-guanylate cyclase output domain. Genes Dev 18: 715-727.

Paul R, Jaeger T, Abel S, Wiederkehr I, Folcher M, Biondi EG, Laub MT, Jenal U. 2008. Allosteric regulation of histidine kinases by their cognate response regulator determines cell fate. Cell 133: 452-461.

Paul K, Nieto V, Carlquist WC, Blair DF, Harshey RM. 2010. The c-di-GMP binding protein YcgR controls flagellar motor direction and speed to affect chemotaxis by a 'backstop brake' mechanism. Mol Cell 38: 128-139.

Paul K, Carlquist WC, Blair DF. 2011. Adjusting the spokes of the flagellar motor with the DNA-binding protein H-NS. J Bacteriol 193: 5914-5922.

Pruggnaller S, Mayr M, Frangakis AS. 2008. A visualization and segmentation toolbox for electron microscopy. J Struct Biol 164: $161-165$.

Quon KC, Marczynski GT, Shapiro L. 1996. Cell cycle control by an essential bacterial two-component signal transduction protein. Cell 84: 83-93.

Rigaut G, Shevchenko A, Rutz B, Wilm M, Mann M, Seraphin B. 1999. A generic protein purification method for protein complex characterization and proteome exploration. Nat Biotechnol 17: 1030-1032.

Ringgaard S, Schirner K, Davis BM, Waldor MK. 2011. A family of ParA-like ATPases promotes cell pole maturation by facilitating polar localization of chemotaxis proteins. Genes Dev 25: 1544-1555.

Ryjenkov DA, Simm R, Romling U, Gomelsky M. 2006. The PilZ domain is a receptor for the second messenger c-diGMP: The PilZ domain protein YcgR controls motility in enterobacteria. I Biol Chem 281: 30310-30314.

Salvetti S, Ghelardi E, Celandroni F, Ceragioli M, Giannessi F, Senesi S. 2007. FlhF, a signal recognition particle-like GTPase, is involved in the regulation of flagellar arrangement, motility behaviour and protein secretion in Bacillus cereus. Microbiology 153: 2541-2552.

Schirmer T, Jenal U. 2009. Structural and mechanistic determinants of c-di-GMP signalling. Nat Rev Microbiol 7: 724-735.

Slaughter BD, Smith SE, Li R. 2009. Symmetry breaking in the life cycle of the budding yeast. Cold Spring Harb Perspect Biol 1: a003384.

St Johnston D, Ahringer J. 2010. Cell polarity in eggs and epithelia: Parallels and diversity. Cell 141: 757-774.

Thanbichler M, Shapiro L. 2006. MipZ, a spatial regulator coordinating chromosome segregation with cell division in Caulobacter. Cell 126: 147-162.

Thompson SR, Wadhams GH, Armitage JP. 2006. The positioning of cytoplasmic protein clusters in bacteria. Proc Natl Acad Sci 103: 8209-8214.

Tsokos CG, Laub MT. 2012. Polarity and cell fate asymmetry in Caulobacter crescentus. Curr Opin Microbiol 15: 744-750.

Viollier PH, Sternheim N, Shapiro L. 2002. A dynamically localized histidine kinase controls the asymmetric distribution of polar pili proteins. EMBO J 21: 4420-4428.

Wolfe AJ, Visick KL. 2008. Get the message out: Cyclic-di-GMP regulates multiple levels of flagellum-based motility. I Bacteriol 190: 463-475.

Yamaichi Y, Bruckner R, Ringgaard S, Moll A, Cameron DE, Briegel A, Jensen GJ, Davis BM, Waldor MK. 2012. A multidomain hub anchors the chromosome segregation and chemotactic machinery to the bacterial pole. Genes Dev 26: 2348-2360. 
Davis et al.

Zarbiv G, Li H, Wolf A, Cecchini G, Caplan SR, Sourjik V, Eisenbach M. 2012. Energy complexes are apparently associated with the switch-motor complex of bacterial flagella. I Mol Biol 416: 192-207.

Zhang Y, Franco M, Ducret A, Mignot T. 2010. A bacterial Raslike small GTP-binding protein and its cognate GAP establish a dynamic spatial polarity axis to control directed motility. PLOS Biol 8: e1000430.

Zheng SQ, Keszthelyi B, Branlund E, Lyle JM, Braunfeld MB, Sedat JW, Agard DA. 2007. UCSF tomography: An integrated software suite for real-time electron microscopic tomographic data collection, alignment, and reconstruction. I Struct Biol 157: 138-147. 


\section{Erratum}

Genes \& Development 27: 2049-2062 (2013)

De- and repolarization mechanism of flagellar morphogenesis during a bacterial cell cycle Nicole J. Davis, Yaniv Cohen, Stefano Sanselicio, Coralie Fumeaux, Shogo Ozaki, Jennifer Luciano, Ricardo C. Guerrero-Ferreira, Elizabeth R. Wright, Urs Jenal, and Patrick.H. Viollier

In the above-mentioned article, Nicole J. Davis and Yaniv Cohen contributed equally. The authors acknowledge there should not be a second notation of equal contribution between Yaniv Cohen and Stefano Sanselicio. 


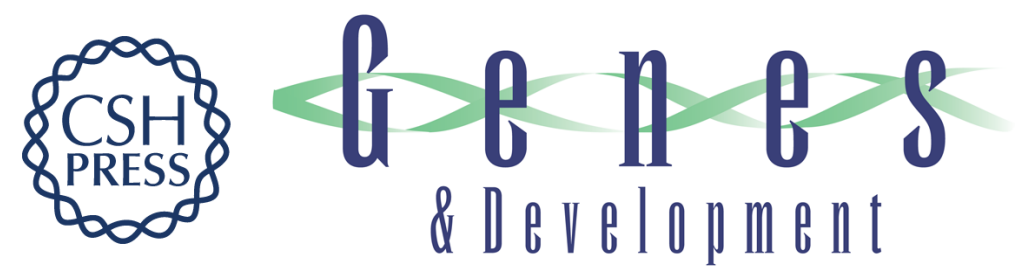

\section{De- and repolarization mechanism of flagellar morphogenesis during a bacterial cell cycle}

Nicole J. Davis, Yaniv Cohen, Stefano Sanselicio, et al.

Genes Dev. 2013, 27:

Access the most recent version at doi:10.1101/gad.222679.113

\section{Supplemental http://genesdev.cshlp.org/content/suppl/2013/09/24/27.18.2049.DC1 \\ Material}

Related Content

De- and repolarization mechanism of flagellar morphogenesis during a bacterial cell cycle

Nicole J. Davis, Yaniv Cohen, Stefano Sanselicio, et al.

Genes Dev. October , 2013 27: 2292

References This article cites 54 articles, 22 of which can be accessed free at:

http://genesdev.cshlp.org/content/27/18/2049.full.html\#ref-list-1

Articles cited in:

http://genesdev.cshlp.org/content/27/18/2049.full.html\#related-urls

Creative This article is distributed exclusively by Cold Spring Harbor Laboratory Press for the first

Commons six months after the full-issue publication date (see

License http://genesdev.cshlp.org/site/misc/terms.xhtml). After six months, it is available under a Creative Commons License (Attribution-NonCommercial 3.0 Unported), as described at http://creativecommons.org/licenses/by-nc/3.0/.

Email Alerting Receive free email alerts when new articles cite this article - sign up in the box at the top Service right corner of the article or click here.

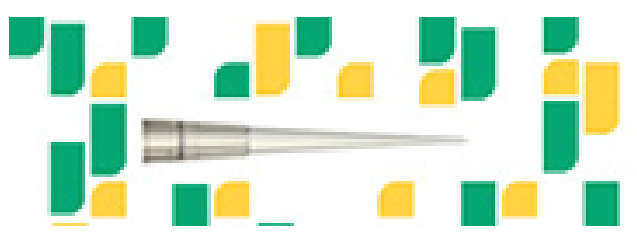

Focused on your science. 